\title{
Interband cascade lasers with short electron injector
}

\author{
Chao Ning (宁 超) $)^{1,2}$, Tian Yu (于 天 $)^{1,2}$ ，Shuman Liu (刘舒曼 $)^{1,2^{*}}$, Jinchuan Zhang (张锦川 $)^{1,2}$ ， Lijun Wang (王利军) $)^{1,2}$ ， \\ Junqi Liu (刘俊岐) $)^{1,2}$, Ning Zhuo (卓 宁 $)^{1,2^{* *}}$, Shenqiang Zhai (翟慎强) $)^{1,2}$, Yuan Li (李 远) $)^{1,2}$, and Fengqi Liu (刘峰奇) $)^{1,2,3}$ \\ ${ }^{1}$ Beijing Key Laboratory of Low Dimensional Semiconductor Materials and Devices, Key Laboratory of Semiconductor Materials Science, \\ Institute of Semiconductors, Chinese Academy of Sciences, Beijing 100083, China \\ ${ }^{2}$ Center of Materials Science and Opto-Electronic Engineering, University of Chinese Academy of Sciences, Beijing 100049, China \\ 3 Beijing Academy of Quantum Information Sciences, Beijing 100193, China
}

*Corresponding author: liusm@semi.ac.cn

${ }^{* *}$ Corresponding author: zhuoning@semi.ac.cn

Received July 17, 2021 | Accepted November 2, 2021 | Posted Online December 6, 2021

\begin{abstract}
We demonstrate GaSb-based interband cascade lasers (ICLs) emitting around $3.65 \mu \mathrm{m}$, which exhibit a room-temperature continuous-wave (CW) output power above $100 \mathrm{~mW}$. Cavity-length analysis showed that the laser structure has a low internal loss of $3 \mathrm{~cm}^{-1}$ while maintaining a total internal quantum efficiency greater than one. After $6400 \mathrm{~h} \mathrm{CW}$ operation at $25^{\circ} \mathrm{C}$, the threshold current of the laser increased by $3 \%$, and the output power decreased by $7 \%$, indicating good reliability of the device.
\end{abstract}

Keywords: interband cascade lasers; quantum well; mid-infrared; semiconductor lasers.

DOI: 10.3788/COL202220.022501

\section{Introduction}

The interband cascade laser $(\mathrm{ICL})^{[1-6]}$ is a compact mid-infrared light source with low threshold current density ${ }^{[7]}$ for many applications such as trace gas sensing ${ }^{[8-11]}$, flame-temperature measurements $^{[12]}$, free-space optical communications ${ }^{[13]}$, infrared lidars, and infrared countermeasures, especially in the wavelength range from $3 \mu \mathrm{m}$ to $4 \mu \mathrm{m}$. An ICL combines the characteristics of the interband optical transition from quantum well (QW) lasers ${ }^{[14]}$ and the electron transport through the cascading stages from quantum cascade lasers ${ }^{[15]}$. In 2008 , Kim et al. reported continuous-wave (CW) lasing at $3.75 \mu \mathrm{m}$ above room temperature $^{[16]}$, which was a milestone in the development of ICLs. At present, the active region of a room-temperature CW ICL includes an active InAs/GaInSb/InAs W-type QW emitting region, with a $\mathrm{GaSb} / \mathrm{AlSb}$ double $\mathrm{QW}$ hole injector on one side and an electron injector formed by an InAs/AlSb chirped superlattice on the other side, in which the InAs wells are n-doped to provide electron injection into the above mentioned active W-type quantum wells.

In a well-known quantum cascade laser, only electrons contribute to the current flow by passing through the conduction subbands in each cascade stage. In contrast, both electrons and holes are injected into the active $\mathrm{W}$ wells by the electron injector and hole injector on both sides, as shown in Fig. 1. Under an appropriate applied bias voltage, a semi-metallic interface (SMIF) forms between the hole injector and the electron injector due to the type II heterojunction between InAs and GaSb. Such an SMIF is essential to ensure the smooth transport of the electrons between stages. If one pays attention to low power consumption, the SMIF should form under a bias voltage as low as possible. The InAs QW on the right side of the SMIF should be thick enough to lower the ground energy level. This electron energy level is lower than the upper lasing energy level in the next stage. Thus, the electron injection is inefficient in earlier ICLs. On the contrary, holes are generated internally from the intrinsically p-type GaSb QWs in the hole injector. The ground energy level of the hole injector lies slightly below the lower lasing energy level in the active GaInSb hole well. From an energy point of view, holes can be injected efficiently into the active $\mathrm{W}$ wells.

In 2011, Vurgaftman et al. ${ }^{[17]}$ proposed a carrier rebalancing scheme, which addressed the issue where the electron injection efficiency in an ICL is much lower than the hole injection efficiency. Four InAs QWs in the middle of the electron injector were heavily doped to inject enough electrons into the active $\mathrm{W}$ wells even though the ground energy level of the electron injector was lower than the upper lasing level of the active $\mathrm{W}$ wells. They reported a reduced threshold current density as low as $167 \mathrm{~A} \cdot \mathrm{cm}^{-2}$ for pulsed operation at room temperature by an optimal doping concentration up to $5 \times 10^{18} \mathrm{~cm}^{-3}$. However, we have noticed in our experiments that such a high doping concentration requires a Si cell temperature higher than $1250^{\circ} \mathrm{C}$. It is challenging to keep the superlattice interfaces smooth and abrupt when the growth surface is irradiated by the high-temperature Si cell. For this reason, we reduced the $\mathrm{Si}$ doping concentration, and, at the same time, we reduced the thicknesses of the InAs wells near the SMIF in the electron 


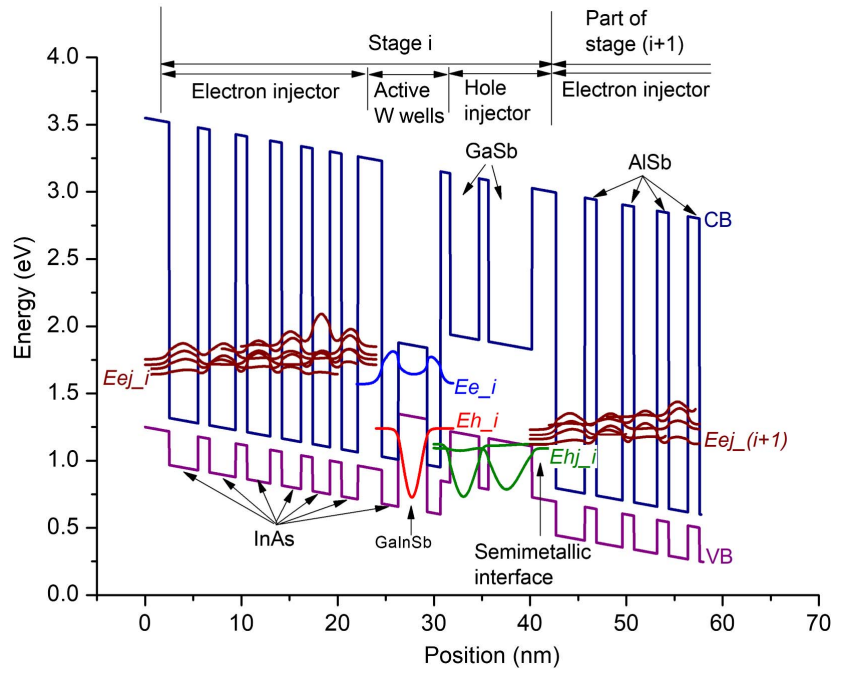

Fig. 1. Energy band diagram of an ICL active stage $i$ composed of an InAS/ Ga. $1 \mathrm{I}_{0.3 \mathrm{~S}} \mathrm{Sb} / \mathrm{InAs}$ W-type active QW sandwiched by an InAs/AlSb chirped superlattice electron injector on the left side and a GaSb/AISb double QW hole injector on the right side. Calculated probability density functions are shown for the upper and lower lasing subbands $E_{e_{i}}$ and $E_{h_{-i} i}$ the hole injection level $E_{h j i i}$ and the electron injection miniband, where the lowest electron injection level is $E_{e j \_i}$. Four InAs QWs in the electron injector of stage $i+1$ are also shown on the right side to illustrate the SMIF for carrier transfer between two sequent stages. The layer structure of the active stage $i$ from the left is as follows: $2.5 \mathrm{~nm}$ AlSb/3.0 nm InAs/1.2 nm AlSb/2.7 nm InAs/1.2 nm AlSb/ $2.4 \mathrm{~nm}$ InAs/1.2 nm AlSb/2.0 nm InAs/1.2 nm AlSb/1.8 nm InAs/1.1 nm AlSb/ $1.7 \mathrm{~nm}$ InAs/2.5 nm AISb/1.7 nm InAs/3.0 nm Ga $0.71 \mathrm{n}_{0.3} \mathrm{Sb} / 1.4 \mathrm{~nm} \mathrm{InAs} / 1.0 \mathrm{~nm}$ AISb/3.0 nm GaSb/1.0 nm AlSb/4.5 nm GaSb/2.5 nm AlSb. Four underlined InAs electron injector QWs were doped with Si to $2 \times 10^{18} \mathrm{~cm}^{-3}$. The bulk conduction band (CB) edge and valence band (VB) edge are indicated by the dark blue and purple lines, respectively.

injector. The reduction of the InAs well thickness causes the lowest energy level of the electron injector to be close to or higher than the upper lasing energy level in the active $\mathrm{W}$ wells. In this way, the electron injection efficiency should be improved even at a lower doping concentration.

In this Letter, we demonstrate an ICL with the Si doping concentration of $2 \times 10^{18} \mathrm{~cm}^{-3}$ accompanied with the narrowed InAs QWs in the electron injector to ensure sufficient electron injection to the active $\mathrm{W}$ wells. The ICL parameters such as the internal quantum efficiency, internal loss, differential gain, and transparent current density of the device were extracted by the cavity-length analysis technique. A room-temperature light output power of more than $100 \mathrm{~mW}$ was achieved from a five-stage ICL with the combination of high reflection (HR) and antireflection (AR) coating on the facets.

\section{Experiments and Methods}

Figure 1 shows the band diagram of the designed ICL active core, which comprises the InAs $/ \mathrm{Ga}_{0.7} \mathrm{In}_{0.3} \mathrm{Sb} / \mathrm{InAs}$ active $\mathrm{W}$-type
QWs of stage $i$, with the chirped InAs/AlSb superlattice electron injector on the left side and the AlSb/GaSb double QW hole injector on the right side. A part of the electron injector from the next stage, i.e., stage $i+1$, is also shown on the right side of the hole injector to illustrate the energy alignment between them. As the InAs electron injector QWs are narrower than that in Ref. [16], the lowest energy level of the electron injector $E_{e j_{-} i}$ is higher than the upper lasing energy level $E_{e_{-} i}$ in stage $i$. At the same time, the lowest injector energy level $E_{e j-(i+1)}$ in stage $i+1$ is aligned with the hole injector subband $E_{h j-i}$ of stage $i$, as indicated in Fig. 1. Therefore, using a relatively low doping concentration of $2 \times 10^{18} \mathrm{~cm}^{-3}$ in the electron injector is expected to ensure an effective electron injection efficiency.

The ICL structure was grown on an epi-ready Te-doped (001) oriented GaSb substrate by the molecular beam epitaxy (MBE) technique. The growth begins with a 500-nm-thick Te-doped $\mathrm{GaSb}$ buffer layer, followed by 610 repetitions of a $2.4 \mathrm{~nm} /$ $2.5 \mathrm{~nm}$ InAs/AlSb superlattice as the bottom cladding layer. Before the growth of AlSb on InAs, an appropriate time of growth interruption is introduced to reduce the As pressure in the MBE chamber and prevent As from being mixed into the subsequent AlSb layer. Next, the active region consisting of five stages of the structure shown in Fig. 1 sandwiched by two 200 -nm-thick GaSb:Te $\left(1 \times 10^{17} \mathrm{~cm}^{-3}\right)$ separate confinement layers (SCLs) was deposited. On top of the upper SCL, 320 repetitions of the InAs/AlSb superlattice structure were grown as the top cladding layer. Finally, the laser structure was ended by a $10-\mathrm{nm}$-thick heavily $\mathrm{n}$-doped InAs top contact.

Both InAs/AlSb superlattice cladding layers were gradually doped by Si. The doping concentration increased from $1 \times 10^{17} \mathrm{~cm}^{-3}$ near the active core to $2 \times 10^{18} \mathrm{~cm}^{-3}$ towards the heavily doped contact layer. Each part of the structure, i.e., the buffer layer, the individual cladding layer, each $\mathrm{GaSb}$ SCL, the active region, and the InAs top contact, was connected by an InAs/AlSb chirped superlattice transition layer to smooth the abrupt conduction band discontinuity between $\mathrm{GaSb}$ and InAs.

The wafer was processed into narrow ridge waveguide devices with stripe widths of $15 \mu \mathrm{m}$ and $20 \mu \mathrm{m}$. The ridge was defined by standard contact photolithography followed by wet etching down through the active region to the bottom GaSb SCL to limit the lateral current diffusion. A 450 -nm-thick $\mathrm{SiO}_{2}$ film was deposited with plasma-enhanced chemical vapor deposition for passivation and electrical insulation. A contact window was opened on top of the ridge by a buffered oxide etching process. Then, a Ti/Pt/Au top contact was deposited by electron beam evaporation, on top of which a 5 - $\mu$ m-thick gold layer was electroplated to improve heat dissipation of the chip. Afterward, a bottom contact of $\mathrm{Ge} / \mathrm{Au} / \mathrm{Pt} / \mathrm{Au}$ was evaporated on the thinned substrate. After rapid annealing, the wafers were cleaved into laser bars of the desired cavity length. The laser bars were mounted epi-side down on copper heat sinks through $\mathrm{In}-\mathrm{Au}$ alloying for characterization. The facets were left uncoated or coated with Au HR film and 1/4 lambda AR film as required. 


\section{Results and Discussion}

The crystalline quality and the lattice mismatch to the substrate of the epilayer were determined by high-resolution X-ray diffraction (XRD) measurements around the GaSb (004) diffraction peak. A scan range of $\pm 10,000$ arcsec was used to ensure that the second-order diffraction peaks from the InAs/AlSb superlattice cladding layer were visible. Figure 2(a) shows the XRD pattern of a sample. The pattern shown below is the result of a dynamic simulation of the entire structure. The strong sharp peaks marked by SL are ascribed to the InAs/AlSb superlattice cladding layer, while the series of weak sharp peaks between the SL peaks comes from the active region. In addition, the inserted chirped superlattice transition layers produce a wide overlapped diffraction pattern as marked by a rectangle. Meanwhile, the InAs cap layer can also be distinguished from the pattern. The separation between the substrate peak and the zeroth-order satellite peak of the epilayer is $80 \mathrm{arcsec}$, indicating a very small lattice mismatch, i.e., less than $0.05 \%$, of the epilayer to the $\mathrm{GaSb}$
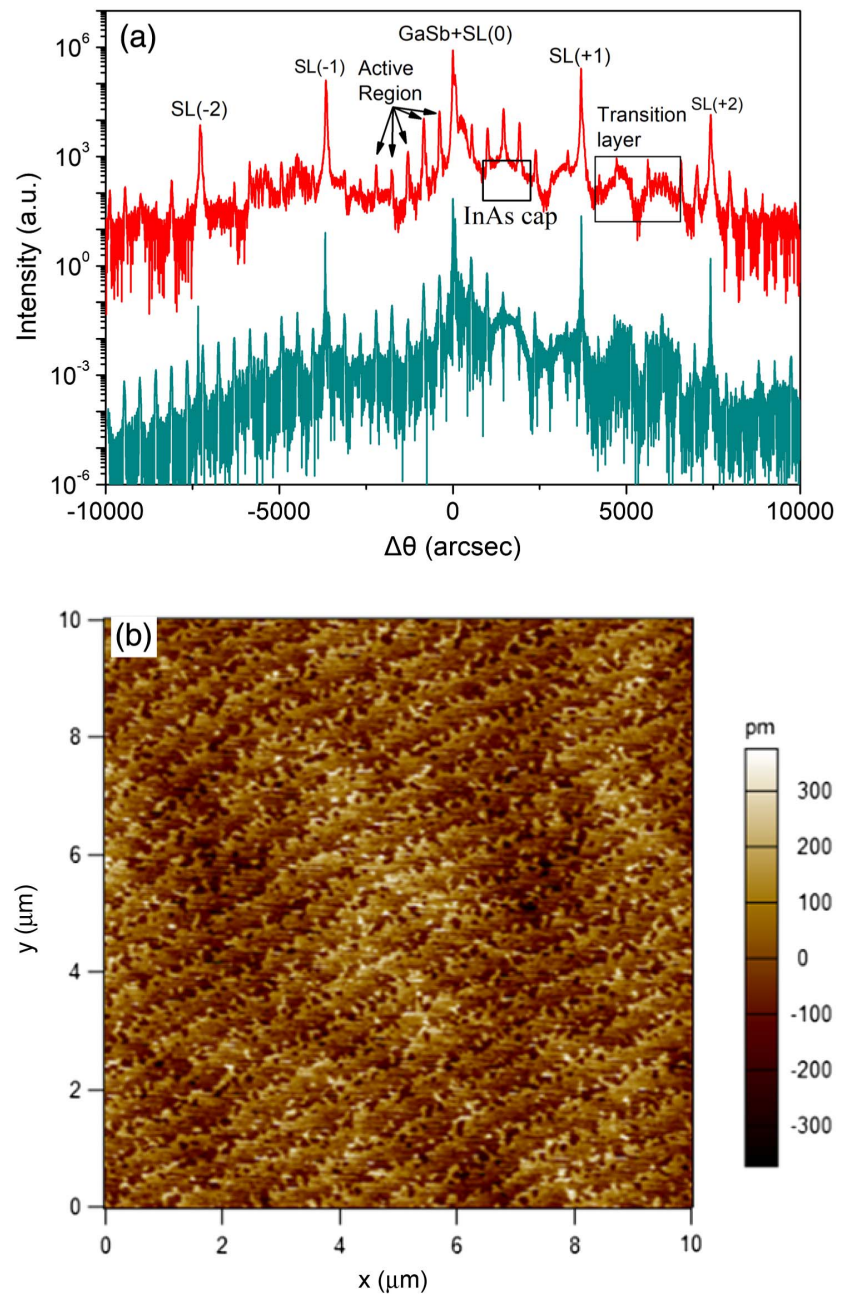

Fig. 2. (a) High-resolution XRD pattern measured around GaSb (004) (top) of the epitaxial complete device structure and the dynamic simulation of the same structure (bottom); (b) AFM image measured on the surface of a complete device structure. substrate. The full width at half-maximum (FWHM) of the firstorder satellite peaks SL $(+1)$ and SL $(-1)$ of the InAs/AlSb superlattices is 20 arcsec, which is close to the FWHM (17 arcsec) of the diffraction peak from the GaSb substrate. The FWHM of the satellite peaks from the active region is 43 arcsec. The RMS roughness of the surface of the $6.1-\mu \mathrm{m}$-thick complete device structure is $0.11 \mathrm{~nm}$, as implied by the atomic force microscope (AFM) image for the $10 \mu \mathrm{m} \times 10 \mu \mathrm{m}$ scan shown in Fig. 2(b). Clear terraces evenly separated by 3 - $\AA$-high atomic steps are visible along the $[-110]$ direction, indicating a step-flux growth mode of the entire ICL structure ${ }^{[18]}$. The overall epitaxial structure has more than 2000 layers of alternately grown InAs, AlSb, $\mathrm{GaSb}$, and $\mathrm{Ga}_{0.7} \mathrm{In}_{0.3} \mathrm{Sb}$, exhibiting high crystal quality and low lattice mismatch to the $\mathrm{GaSb}$ substrate.

The CW lasing spectra of a laser with both facets as-cleaved at various temperatures are shown in Fig. 3. The ridge was $20 \mu \mathrm{m}$ wide and $3 \mathrm{~mm}$ long. Each spectrum exhibits a typical multipeak feature of a Fabry-Pérot cavity. The emission wavelengths varied from $3.6 \mu \mathrm{m}$ at $10^{\circ} \mathrm{C}$ to $3.75 \mu \mathrm{m}$ at $50^{\circ} \mathrm{C}$, and the red-shift rate is about $3.7 \mathrm{~nm} / \mathrm{deg}$.

To characterize the laser in detail, we carried out the cavitylength analysis on the lasers. Light-current-voltage $(L-I-V)$ measurements were conducted at two heat sink temperatures of $10^{\circ} \mathrm{C}$ and $20^{\circ} \mathrm{C}$ on the as-cleaved laser dies having a ridge width of $20 \mu \mathrm{m}$ and different cavity lengths from $2 \mathrm{~mm}$ to $5 \mathrm{~mm}$. The power slope efficiency $\mathrm{d} P / \mathrm{d} I$ above the threshold, which is proportional to the external quantum efficiency $\eta_{\text {ext }}$, and the threshold current density $J_{\text {th }}$ were obtained from the $L-I-V$ measurements. The laser parameters are deduced from plots of $1 / \eta_{\text {ext }}$ versus cavity length $L$ and $J_{\text {th }}$ versus inverse cavity length $1 / L$ according to Eqs. (1) and (2):

$$
\frac{1}{\eta_{\mathrm{ext}}}=\frac{h v}{q} \frac{1}{\mathrm{~d} P}=\frac{1}{\eta_{i} I}+\frac{1}{\eta_{i}} \frac{a_{i}}{a_{m}}=\frac{1}{\eta_{i}}+\frac{1}{\eta_{i}} \frac{2 a_{i}}{\ln \left(\frac{1}{R_{1} R_{2}}\right)} L,
$$

$$
J_{\text {th }}=\frac{q}{\eta_{i} \tau}\left[n_{\text {tr }}+\frac{a_{i}+a_{m}}{\Gamma(\mathrm{d} g / \mathrm{d} n)}\right]=\left(J_{\mathrm{tr}}+\frac{a_{i}}{g_{d}}\right)+\left(\frac{1}{2 g_{d} L}\right) \ln \left(\frac{1}{R_{1} R_{2}}\right),
$$

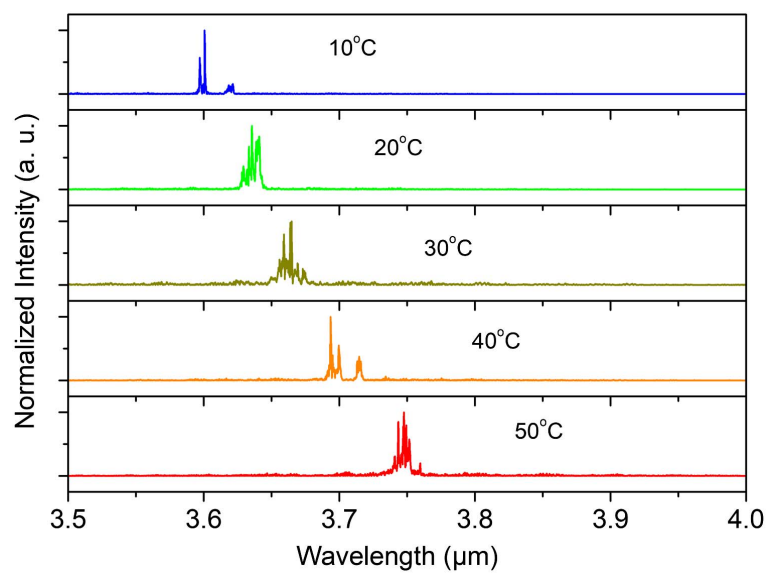

Fig. 3. Lasing spectra of the ICL at various temperatures. 
where $h$ is the Planck constant, $v$ is the lasing frequency, $\eta_{i}$ is the overall internal quantum efficiency, $a_{i}$ is the internal loss, $a_{m}$ is the mirror loss, $R_{1}$ and $R_{2}$ are the reflectivities of the front and rear facets of the laser, which are 0.31 for the cleaved facets, $\tau$ is the carrier lifetime, $n_{\text {tr }}$ is the transparency carrier density, $J_{\text {tr }}$ is the transparency current density, and $g_{d}$ is the peak modal differential gain.

Figure 4 shows the results of cavity-length analysis of the uncoated ICLs at the heat sink temperatures of $10^{\circ} \mathrm{C}$ and $20^{\circ} \mathrm{C}$. The points in Fig. 4(a) show $1 / \eta_{\text {ext }}$ as a function of the cavity length $L$. The lines represent fits to Eq. (1), yielding the overall internal quantum efficiency $\eta_{i}=1.51$ and 1.29 at $T=10^{\circ} \mathrm{C}$ and $20^{\circ} \mathrm{C}$, respectively, and $a_{i}=3 \mathrm{~cm}^{-1}$ for both cases. The value of $\eta_{i}$ exceeds unity even though it is strongly dependent on the temperature. As we know, once the design is optimized, the overall internal quantum efficiency greater than one is a feature of a cascade laser since it is the single-stage internal quantum efficiency multiplied by the number of stages. Moreover, the internal loss of our device is lower than that reported in Refs. $[19,20]$.

The threshold current densities $J_{\text {th }}$ of the ICLs with different cavity lengths measured at $10^{\circ} \mathrm{C}$ and $20^{\circ} \mathrm{C}$ are shown in Fig. 4(b). By fitting $J_{\text {th }}$ as a function of $1 / L$ according to Eq. (2), we
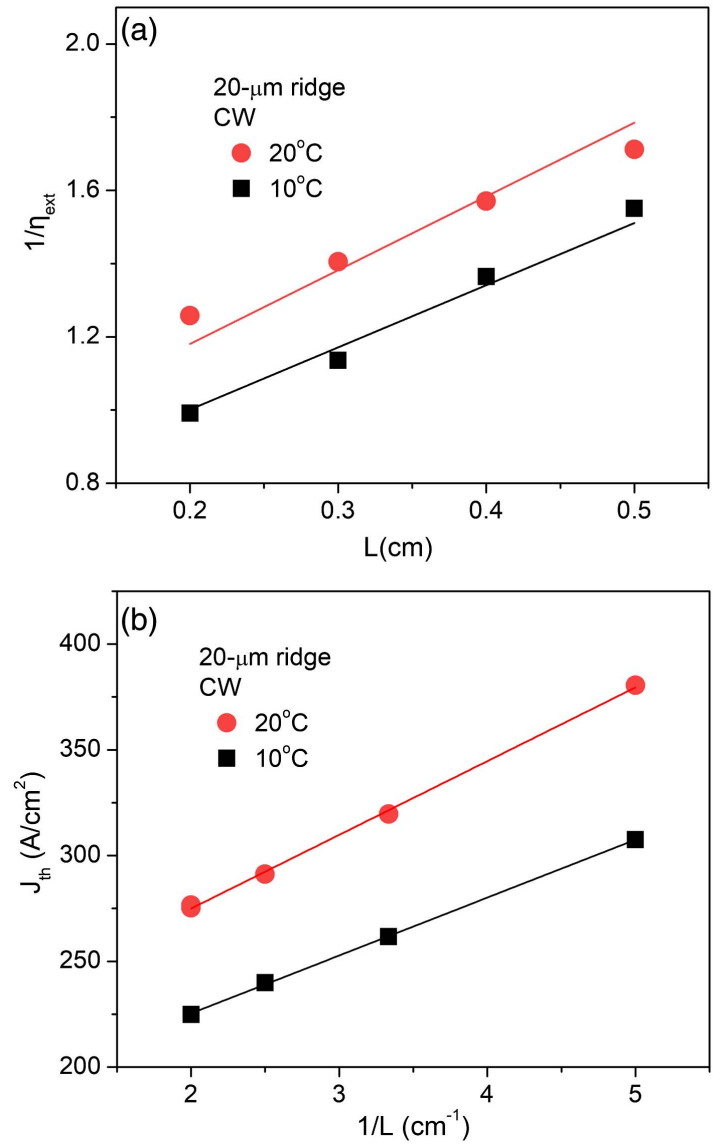

Fig. 4. (a) Measured inverse of the external quantum efficiency $1 / \eta_{\text {ext }}$ as a function of cavity length $L$ and (b) threshold current density $J_{\text {th }}$ as a function of the reciprocal of cavity length $1 / L$. Values of internal quantum efficiency, internal loss, peak modal differential gain, and transparency current density are obtained by fitting the data with Eqs. (1) and (2). obtained $g_{d}=0.0418 \mathrm{~cm} / \mathrm{A}$ and $J_{\mathrm{tr}}=94 \mathrm{~A} / \mathrm{cm}^{2}$ at $T=10^{\circ} \mathrm{C}$ and $g_{d}=0.0314 \mathrm{~cm} / \mathrm{A}$ and $J_{\text {tr }}=102 \mathrm{~A} / \mathrm{cm}^{2}$ at $T=20^{\circ} \mathrm{C}$. It can be seen that $g_{d}$ and $J_{\text {tr }}$ are temperature dependent. Since our CW characteristics are affected significantly by thermal effects, these two parameters are degraded markedly even if the heat sink temperature is increased by only $10^{\circ} \mathrm{C}$, indicating that Auger recombination dominates the threshold current ${ }^{[20]}$.

Figure 5 shows the CW $L-I-V$ curves of a laser with $15 \mu \mathrm{m}$ ridge width and $5 \mathrm{~mm}$ cavity length at various temperatures between $10^{\circ} \mathrm{C}$ and $60^{\circ} \mathrm{C}$. The front and rear facets were $\mathrm{AR}$ and $\mathrm{HR}$ coated, respectively. We achieved a CW output power of $126 \mathrm{~mW}$ at $10^{\circ} \mathrm{C}$, which is consistent with the high $\eta_{i}$ and low $a_{i}$.

In order to verify the effect of HR/AR coating on the device performance, we compared the temperature-dependent threshold current density and slope efficiency $\mathrm{d} P / \mathrm{d} I$ of the HR/AR sample with an uncoated (UN/UN) laser. The results are displayed in Fig. 6. Because an HR coating reduces while an AR coating increases the mirror loss, the total mirror loss should be close to the UN/UN sample if the reflectivity of the HR coating is high enough. That is to say, the threshold current density should not be affected by HR/AR coating significantly. It can be seen from Fig. 6(a) that the threshold current density of the HR/AR sample is slightly lower than that of the UN/UN sample at all temperatures.

On the other hand, the main function of the HR coating on the rear facet is to collect the light output power of the active region from the front facet completely, that is, the output power should be twice that of the UN/UN sample. Figure 6(b) plots the differential slope efficiency $\mathrm{d} P / \mathrm{d} I$ of the HR/AR sample with that of the UN/UN sample as a function of temperature. For a more intuitive comparison, $\mathrm{d} P / \mathrm{d} I$ from double facets of the UN/UN sample is also shown in Fig. 6(b). This set of data almost coincides with the HR/AR sample, that is, the reflectivity of the HR coating is very close to one as expected.

All of the applications of ICLs require high reliability and a long lifetime $e^{[21-23]}$. We have recently carried out an aging study of a representative ICL sample. The laser with a ridge

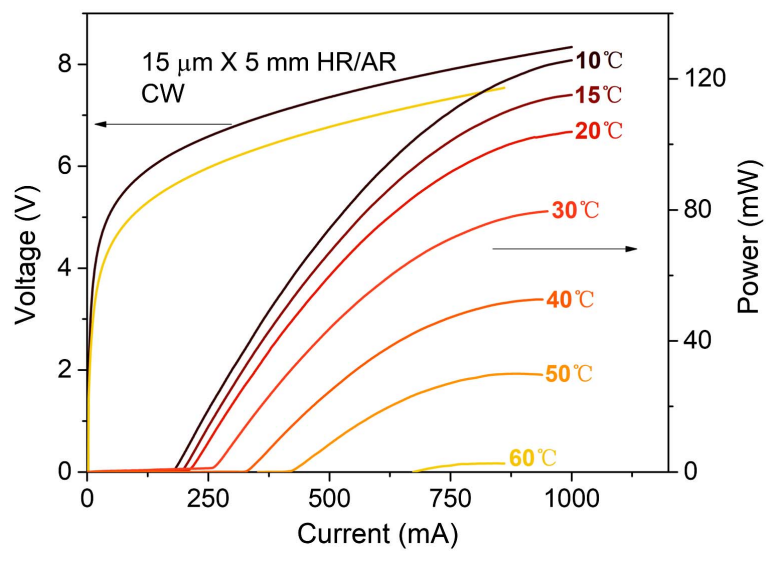

Fig. 5. CW L-I-V characteristics for a 15- $\mu$ m-wide, 5-mm-long device with HR and $A R$ coating at various heat-sink temperatures between $10^{\circ} \mathrm{C}$ and $60^{\circ} \mathrm{C}$. 

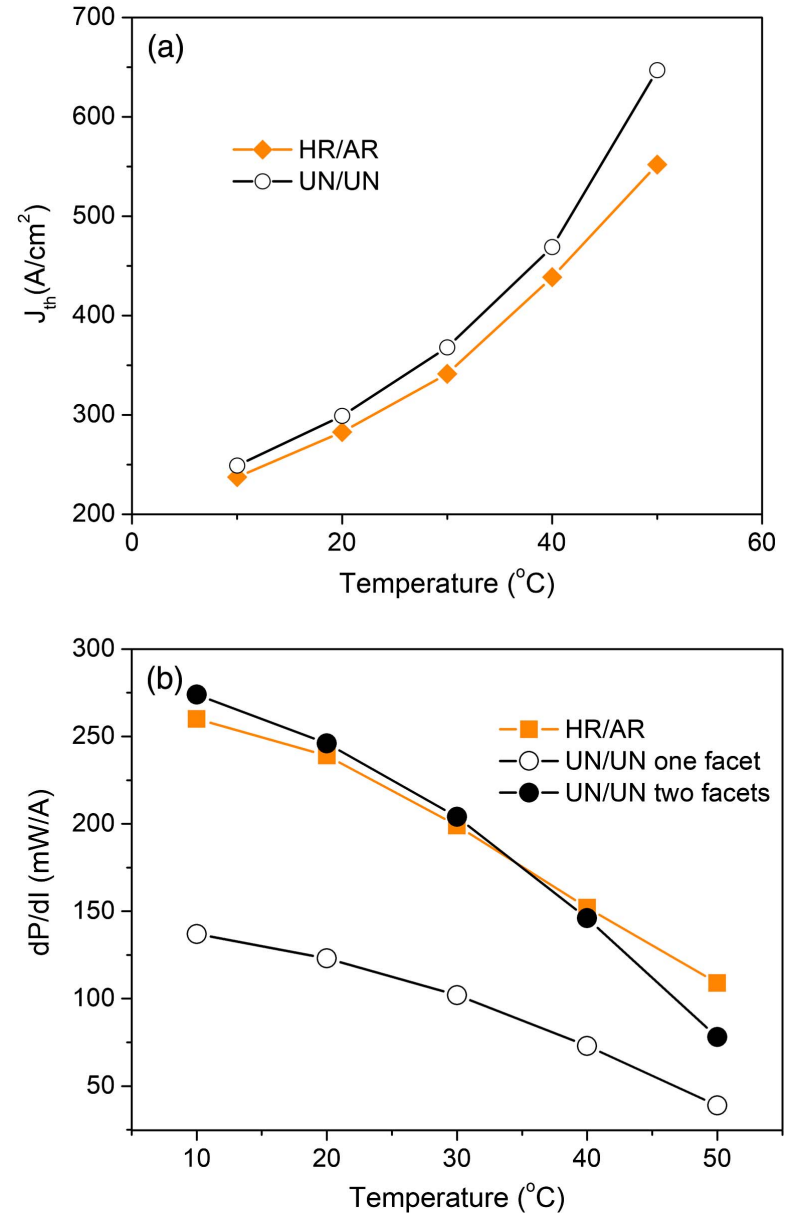

Fig. 6. (a) CW threshold current densities and (b) differential slope efficiencies versus temperature for an HR/AR and an UN/UN samples.

width of $15 \mu \mathrm{m}$ and cavity length of $3 \mathrm{~mm}$ was soldered epitaxialside down on a $\mathrm{Cu}$ heat sink. The aging test was performed at $25^{\circ} \mathrm{C}$ with a CW injection current of $360 \mathrm{~mA}$, which is two times the threshold current. The emitted power was around $40 \mathrm{~mW}$. The output power was recorded every hour. Figure 7(a) shows the monitoring results of the $6400 \mathrm{~h} \mathrm{CW}$ operation. The device exhibited little degradation in output power during the $6400 \mathrm{~h}$ test.

The small fluctuations shown in Fig. 7(a) were mainly caused by the current fluctuation of the homemade power supply used in this test. This output power degradation test is still ongoing. In addition, the $L-I-V$ curves measured before the aging test $(0 \mathrm{~h})$ and after the CW operation for 3500, 3900, 4700, and $6400 \mathrm{~h}$ are shown in Fig. 7(b). After 6400 h of CW operation, the threshold current density of the laser just increased by $2 \%$, and the maximum output power reduced by $7 \%$. It is well known that the degradation of the output power is usually caused by the generation of defects in a laser structure. As mentioned previously, our ICL structure has more than 2000 layers, resulting in a total thickness greater than $6 \mu \mathrm{m}$. Thus, the small amount of degradation of our device, i.e., the good long-term stability, indicates the high-quality epitaxial structures.
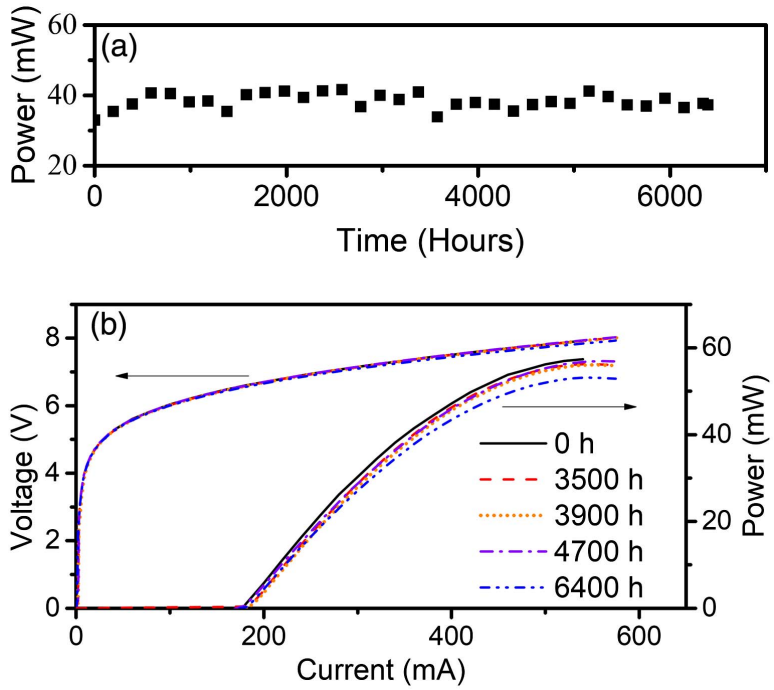

Fig. 7. (a) CW output power of a $15 \mu \mathrm{m} \times 3 \mathrm{~mm}$ ICL. The data were recorded every hour. (b) L-I-V characteristics for the ICL measured before the lifetime test ( $0 \mathrm{~h}$ ) and after CW operation for $3500 \mathrm{~h}, 3900 \mathrm{~h}, 4700 \mathrm{~h}$, and $6400 \mathrm{~h}$.

\section{Summary}

In summary, we produced ICLs that exhibited CW room-temperature output power above $100 \mathrm{~mW}$ with HR/AR coated facets. A high internal quantum efficiency of 1.51 and a low internal loss of $3 \mathrm{~cm}^{-1}$ were achieved by thinning the electron injector and decreasing the doping concentration. Furthermore, our ongoing aging experiments revealed that the threshold current density remains almost constant for a long time at a heat sink temperature of $25^{\circ} \mathrm{C}$, indicating that our devices have good reliability.

\section{Acknowledgement}

This work was supported by the National Key Research and Development Project (No. 2018YFB2200500) and the National Natural Science Foundation of China (Nos. 61790583 and 61774150).

\section{References}

1. J. R. Meyer, W. W. Bewley, C. L. Canedy, C. S. Kim, M. Kim, C. D. Merritt, and I. Vurgaftman, “The interband cascade laser,” Photonics 7, 75 (2020).

2. R. Q. Yang, L. Li, W. Huang, S. M. S. Rassel, J. A. Gupta, A. Bezinger, X. Wu, S. G. Razavipour, and G. C. Aers, "InAs-based interband cascade lasers," IEEE J. Sel. Top. Quantum Electron. 25, 1200108 (2019).

3. T. Yu, S. M. Liu, J. C. Zhang, B. Xu, L. J. Wang, J. Q. Liu, N. Zhuo, S. Q. Zhai, X. L. Ye, Y. H. Chen, F. Q. Liu, and Z. G. Wang, "InAs-based interband cascade lasers at $4.0 \mu \mathrm{m}$ operating at room temperature," J. Semicond. 39, 114003 (2018).

4. C.-H. Lin, W. Y. Hwang, H. Q. Le, Y. Mu, A. Liu, J. Zheng, A. Delaney, C. H. Kuo, and S. S. Pei, "Interband cascade lasers," Proc. SPIE 3947, 122 (2000).

5. I. Vurgaftman, C. L. Canedy, C. S. Kim, M. Kim, W. W. Bewley, J. R. Lindle, J. Abell, and J. R. Meyer, "Mid-infrared interband cascade lasers operating at ambient temperatures,” New J. Phys. 11, 125015 (2009). 
6. A. Bauer, M. Dallner, M. Kamp, S. Höfling, L. Worschech, and A. Forchel, "Shortened injector interband cascade lasers for 3.3- to 3.6- $\mu \mathrm{m}$ emission," Opt. Eng. 49, 111117 (2010).

7. R. Weih, M. Kamp, and S. Höfling, "Interband cascade lasers with room temperature threshold current densities below $100 \mathrm{~A} / \mathrm{cm}^{2}$," Appl. Phys. Lett. 102, 231123 (2013).

8. J. Scheuermann, P. Kluczynski, K. Siembab, M. Straszewski, J. Kaczmarek, R. Weih, M. Fischer, J. Koeth, A. Schade, and S. Höfling, "Interband cascade laser arrays for simultaneous and selective analysis of C1-C5 hydrocarbons in petrochemical industry," Appl. Spectrosc. 75, 336 (2021).

9. Z. Liu, C. Zheng, C. Chen, Y. Li, H. Xie, Q. Ren, Y. Wang, and F. K. Tittel, "ICL-based mid-infrared carbon dioxide sensor system for deep-sea natural gas hydrate exploration," Opt. Express 27, 5598 (2019).

10. R. Ghorbani and F. M. Schmidt, "ICL-based TDLAS sensor for real-time breath gas analysis of carbon monoxide isotopes," Opt. Express 25, 12743 (2017).

11. H. Zheng, Y. Liu, H. Lin, R. Kan, P. Patimisco, A. Sampaolo, M. Giglio, W. Zhu, J. Yu, F. K. Tittel, V. Spagnolo, and Z. Chen, "Sub-ppb-level $\mathrm{CH}_{4}$ detection by exploiting a low-noise differential photoacoustic resonator with a room-temperature interband cascade laser," Opt. Express 28, 19446 (2020).

12. D. Wen and Y. Wang, "Spatially and temporally resolved temperature measurements in counterflow flames using a single interband cascade laser," Opt. Express 28, 37879 (2020).

13. A. Soibel, M. W. Wright, W. H. Farr, S. A. Keo, C. J. Hill, R. Q. Yang, and H. C. Liu, "Midinfrared interband cascade laser for free space optical communication," IEEE Photon. Technol. Lett. 22, 121 (2010).

14. H. Hu, J. Zhao, W. Wang, J. Ho, L. Kuang, and W. Liu, "12 W high power InGaAsP/AlGaInP 755 nm quantum well laser," Chin. Opt. Lett. 17, 061403 (2019).
15. J. Faist, F. Capasso, D. L. Sivco, C. Sirtori, A. L. Hutchinson, and A. Y. Cho, "Quantum cascade laser," Science 264, 553 (1994).

16. M. Kim, C. L. Canedy, W. W. Bewley, C. S. Kim, J. R. Lindle, J. Abell, I. Vurgaftman, and J. R. Meyer, "Interband cascade laser emitting at $\lambda=3.75 \mu \mathrm{m}$ in continuous wave above room temperature," Appl. Phys. Lett. 92, 191110 (2008).

17. I. Vurgaftman, W. W. Bewley, C. L. Canedy, C. S. Kim, M. Kim, C. D. Merritt, J. Abell, J. R. Lindle, and J. R. Meyer, "Rebalancing of internally generated carriers for mid-infrared interband cascade lasers with very low power consumption," Nat. Commun. 2, 585 (2011).

18. T. Yu, S. M. Liu, X. L. Ye, N. Zhuo, F. Q. Liu, B. Xu, and Z. G. Wang, "Defect formation and elimination during the growth of GaSb epilayer," J. Nanosci. Nanotechnol. 18, 7455 (2018).

19. G. Ryu, A. N. Chryssis, J. Amirloo, S. Saini, F. J. Towner, and M. Dagenais, "Gain and losses and room-temperature operation in interband cascade lasers," IEEE Photon. 4, 133 (2012).

20. R. P. Leavitt, J. D. Bruno, J. L. Bradshaw, K. M. Lascola, J. T. Pham, F. J. Towner, S. Suchalkin, G. Belenky, I. Vurgaftman, C. L. Canedy, W. W. Bewley, C. S. Kim, M. Kim, C. D. Merritt, and J. R. Meyer, "High performance interband cascade lasers at 3.8 microns," Proc. SPIE 8277, 82771E (2012).

21. X. Liu, M. Liu, Y. Wang, K. Huang, M. Lei, W. Liu, and Z. Wei, "Mode-locked all-fiber laser with high stability based on cobalt oxyfluoride," Chin. Opt. Lett. 19, 081902 (2021).

22. H. Nie, F. Wang, J. Liu, K. Yang, B. Zhang, and J. He, "Rare-earth ions-doped mid-infrared $(2.7-3 \mu \mathrm{m})$ bulk lasers: a review [Invited]," Chin. Opt. Lett. 19, 091407 (2021).

23. Z. Qu, P. Lu, Y. Li, X. Fu, W. Zhang, D. Liu, and J. Zhang, "Low-frequency acoustic Fabry-Pérot fiber sensor based on a micromachined silicon nitride membrane," Chin. Opt. Lett. 18, 101201 (2020). 